\title{
Comprehensive Review of Golgi Staining Methods for Nervous Tissue
}

\author{
Hee Won Kang ${ }^{1}$, Ho Kyu Kim ${ }^{1}$, Bae Hun Moon ${ }^{1}$, Seo Jun Lee ${ }^{1}$, Se Jung Lee ${ }^{2}$, Im Joo Rhyu ${ }^{2,3, *}$ \\ ${ }^{1}$ Department of Medicine, Korea University College of Medicine, Seoul 02841, Korea \\ ${ }^{2}$ Department of Anatomy, Korea University College of Medicine, Seoul 02841, Korea \\ ${ }^{3}$ Division of Brain Korea 21 Plus Program for Biomedical Science, Korea University College of Medicine, Seoul 02841, Korea
}

*Correspondence to:

Rhyu IJ,

Tel: +82-2-2286-1149

Fax: +82-2-929-5696

E-mail: irhyu@korea.ac.kr

Received June 22, 2017

Revised June 30, 2017

Accepted June 30, 2017
Golgi staining has been modified and developed since Camillo Golgi introduced the black reaction in 1873. This study focuses on the commonly used Golgi staining methods and presents comprehensive data regarding three Golgi staining methods along with their strong and weak points. The Golgi-Cox method uses mercuric chloride for brain tissue impregnation and is a reliable technique for analyzing the complete dendritic tree of cortical neurons. However, specimens tend to shrink during the staining steps. Recent combination of the Golgi-Cox method and immunofluorescence provides additional options for neuroscientists. Rapid Golgi staining requires osmium tetroxide for the postfixation process. It homogenously stains whole structures of neurons and provides their detailed anatomical morphology. This staining is influenced by the age of the specimen, temperature of the laboratory, and duration of each procedure. The Golgi-Kopsch method uses formaldehyde and glutaraldehyde instead of osmium tetroxide and can be used regardless of the age of the specimen and the duration after fixation. This method is suitable for research using human brain fixed for a long time or for specimens obtained from old-aged animals. Selecting a Golgi staining protocol that is appropriate for the specimen type and research purpose is important to achieve best results.

Key Words: Golgi, Rapid Golgi, Golgi Cox, Golgi Kopsch, Modified Golgi method

\section{INTRODUCTION}

\section{History of Golgi Staining}

Since Camillo Golgi and Santiago Ramon y Cajal were awarded the Nobel Prize in 1906, neuroscience has rapidly developed for 111 years because of their contributions. Golgi (1873) developed an extraordinary staining method that uses silver nitrate impregnation of brain tissues for visualizing neurons. The method was initially called the black reaction and was later officially termed Golgi staining. Golgi first investigated Golgi-stained cerebellar tissues and expanded the research to other brain parts (Bentivoglio et al., 2011). Cajal modified this method to develop a more predictable technique and provided extensive data regarding the anatomy of neural tissues. He also performed staining of various specimens that were systemically classified with regard to age and species, thereby making him the father of modern neuroscience.

The relationship between these two great neuroscientists was close but paradoxical in that their scientific theories differed. Cell theory, which was applied to the scientific field at the end of the 19th century, was considered to be an exception to brain tissues before the research by Cajal, and the reticular theory was established as mainstream (De Carlos \& Borrell, 2007). In other words, many scientists supposed that the brain was a well-organized network and not a large aggregation of cells. Although Golgi adhered to this theory, Cajal proposed a progressive interpretation on the basis of the neuronal theory, which focused on each cell in the brain tissue, and denied the reticular theory. This made him a pioneer of the neuronal

(a) This is an open-access article distributed under the terms of the Creative Commons Attribution Non-Commercial License (http://creativecommons.org/licenses/by-nc/4.0) which permits unrestricted noncommercial use, distribution, and reproduction in any medium, provided the original work is properly cited.

Copyrights @ 2017 by Korean Society of Microscopy 
Kang HW et al.

theory, which has since inspired numerous scientists. Because of the error in Golgi persisting with the reticular theory, some researchers now emphasize that Cajal established the cornerstone of the neuronal theory, although others insist that Golgi's invention of the staining method should not be underestimated (Glickstein, 2006; Jones, 2011; Pannese, 2007). Cajal acquired tremendous cytological data using Golgi staining and also acknowledged the excellence of the staining method. Publications that used Golgi staining for the research steadily increased up to the mid-1900s (Pannese, 1999). This staining method was the sole technique that could visualize neurons at that time. On the basis of this technological background, more modified and developed staining methods, such as Golgi-Cox, rapid Golgi, and Golgi-Kopsch staining, were invented and extensively used in research of various specimens (Maiti et al., 2015). Thus, there are approximately 3000 publications that used Golgi staining between 1965 and 1999. While Golgi staining is continues to be used as a central technique, it has been combined with fluorescent staining (Koyama \& Tohyama, 2013; Spiga et al., 2011). In addition, the latest developments in 3D Edge microscopy provide a solution to overcome the limitation of focal depth during the analysis of thick specimens (Rhyu et al., 2016). Golgi staining has contributed in investigating neuronal cytology and the circuitry and connectivity of brain tissues. Neuroscientists can thoroughly probe the fine morphology of neurons, including the soma, axon, dendrites, and dendritic spines.

In the last 40 years, immunostaining techniques at the molecular level have been extensively used for research on nervous tissues. However, Golgi staining has some advantages that cannot be achieved with immunostaining. First, it is impossible to immunostain neurons if there is no specific antibody. Golgi staining remains highly valued for morphological studies because there are countless proteins that cannot be targeted with specific antibodies. Second, there is a limitation to immunostaining if the specimen is a human brain tissue because in such specimens, protein denaturation inevitably occurs, decreasing the specific labeling of neurons. However, Golgi staining can overcome these limitations because it stains neurons randomly and completely. Finally, the cytoarchitectural data derived from Golgi staining can be a guideline for analyzing immunostaining results (Valverde, 1970). This study focuses on commonly used Golgi staining methods, and presents and compares comprehensive data regarding the three Golgi staining techniques. Researchers can select an appropriate method according to the specific goals of their research.

\section{INTRODUCTION TO THREE TYPES OF GOLGI STAINING}

\section{Golgi-Cox Method}

The Golgi-Cox method was first described by Cox in 1891 and continues to be widely used among other Golgi modifications. In this method, potassium dichromate and potassium chloride are used in the impregnation steps, similar to other methods; however, mercuric chloride can also be added to this method, which is distinct from the other methods. Golgi-Cox staining comprises the following three steps: specimen impregnation, tissue protection, and color development. The Golgi-Cox method is considered to be one of the most reliable techniques for demonstrating dendritic arborization, with a low interference level from background structures (Buell, 1982; Koyama, 2013). In addition, the method has the advantage in staining various types of neurons compared with other staining methods, and the entire dendritic tree of cortical neurons can be clearly visualized (Rosoklija et al., 2003). However, as with the other staining methods, there is the disadvantage that neurons are randomly stained. To enhance the specificity of staining, a method that combined immunofluorescence and Golgi staining was recently developed (Spiga et al., 2011).

\section{Rapid Golgi Method}

In 1933, Cajal and De Castro developed the rapid Golgi method that involved the use of osmium tetroxide as a postfixation process to supplement the original Golgi stain, as well as other staining methods used in the early days. This rapid Golgi method reduced the time required for performing the original staining method, i.e., from several weeks to days (Ramon-Moliner, 1970; Scheibel \& Scheibel, 1978; Valverde, 1970). The method also has another advantage of enabling the visualization of anatomical features in neurons. It can homogeneously stain neural cell structures such as axons, cell bodies, dendrites, and dendritic spines, so that morphologic details can be clearly visualized. However, its disadvantage is that the reproducibility of the experiments is unpredictable. To improve this, sectioning with a vibratome could be performed after fixation, enabling the staining resolution to be increased (Patro et al., 2013).

\section{Golgi-Kopsch Method}

The Golgi-Kopsch method, also called aldehyde Golgi or Colonnier-Golgi, is a technique developed by Kopsch and Colonnier using formaldehyde and glutaraldehyde instead of osmium tetroxide, which is used in the rapid Golgi method. The Golgi-Kopsch method has a great advantage in that all areas of the animal brain can be used as specimens for Golgi staining, regardless of the age of the animals (Riley, 1979). The other Golgi staining methods, such as the Golgi-Cox 
and rapid Golgi methods, have a limitation that their use for some subcortical areas of specimens or specimens from oldaged animals is difficult (Koyama, 2013). Moreover, with the Golgi-Kopsch method, brain specimens can be stained even if several years have passed since the specimens were first fixed and can also be used immediately after perfusion. However, the impregnation intensity of neurons is weak compared with that of the rapid Golgi and Golgi Cox methods. To solve this problem, Triton X-100 (polyoxyethylene-10-octylphenylether) is added to facilitate the chemical reactions (Tokuno et al., 1990).

\section{SPECIFIC POINTS REGARDING EACH METHOD}

\section{Golgi-Cox Method}

As mentioned above, impregnation, tissue protection, and color development are the main steps of the Golgi-Cox method, with additional sectioning and mounting steps. Detailed procedures are described in "Golgi-Cox Staining Step by Step" (Zaqout \& Kaindl, 2016). In the impregnation step, silver nitrate is replaced with mercury chloride, and similar to the other methods, potassium dichromate and potassium chromate are used in this step. Solution-treated specimens are kept in the dark for 7 10 days because with longer impregnation, brain sections were found to be less suitable for morphometric evaluation owing to more stain precipitation and artifacts (Narayanan et al., 2014). In the next step, i.e., tissue protection, two solutions are used: a buffer solution and a cryoprotectant solution containing sucrose and polyvinylpyrrolidone. The specimen is stored in a dark room at $4^{\circ} \mathrm{C}$ for approximately $4 \sim 7$ days. Next, the sectioning step is performed using a vibratome.

In the color development process, the specimen is dehydrated and developed. Dehydration using ethanol and cleaning with xylol are repeated many times, resulting in further quality improvement (Zaqout \& Kaindl, 2016). The specimens are then mounted and observed.

\section{Rapid Golgi}

The rapid Golgi method comprises the pre-fixation, rinsing, post-fixation, staining, and embedding processes (Smitha \& Roopa, 2012). Detailed procedures are described in "The Methods of Golgi” (Scheibel \& Scheibel, 1978). There are some points that need to be noted regarding this method. Selecting this method is better if the research purpose is analyzing the cerebral cortex because the cerebral cortex is stained more clearly than the other brain parts in the rapid Golgi method. Furthermore, if the specimen is younger, the target is stained more clearly. To reduce errors in the
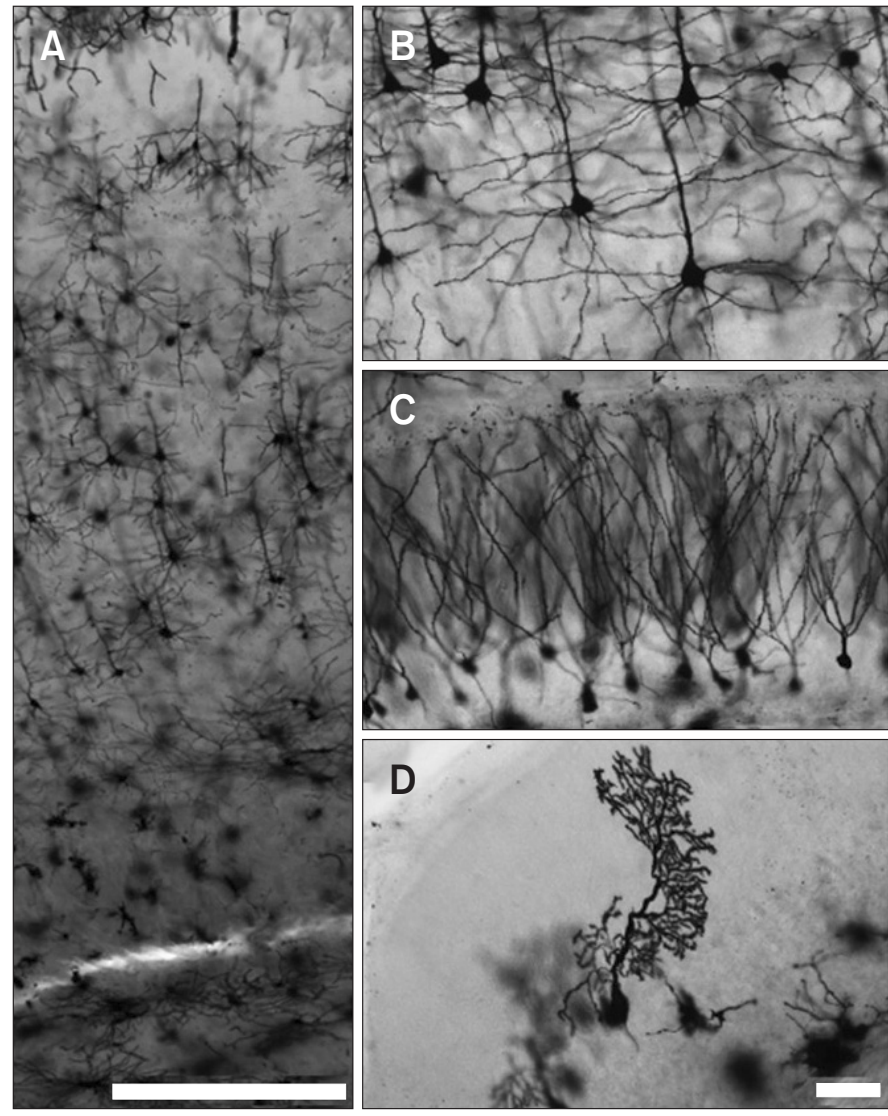

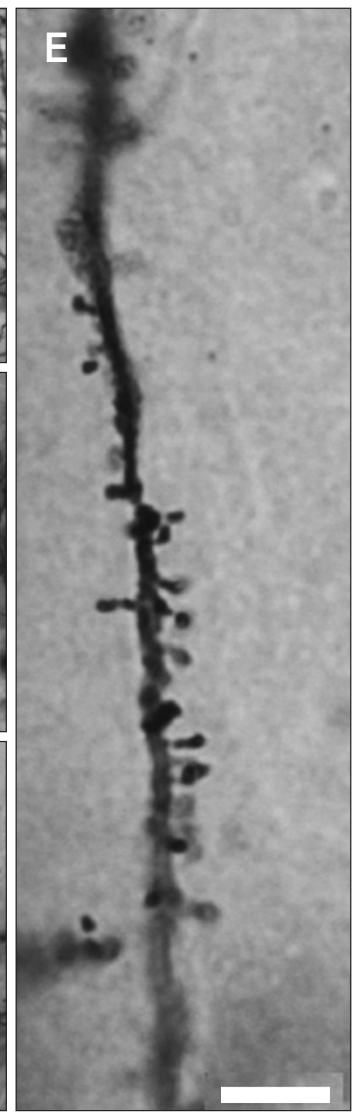

Fig. 1. Adult mouse brain specimen prepared using the Golgi-Cox method (Modified from Zaqout \& Kaindl, 2016 with permission based on the open access policy). Magnified images of the cerebral cortex (A, B), hippocampus (C), and cerebellar cortex (D). Dendritic spines can also be visualized at a higher magnification (E). 
procedures, the laboratory temperature should not be $<10^{\circ} \mathrm{C}$ during the staining procedure. While manufacturing blocks for sectioning, it is favorable to complete Epon processing as quickly as possible and immerse the specimens in silver nitrate for $<5$ days. After covering with balsam and cover slipping, specimen observations should be completed within 3 months, otherwise, the specimens begin to de-impregnate (Ahn et al., 1990).

\section{Golgi-Kopsch Method}

The Golgi-Kopsch method is similar to the Golgi-Cox method, but it is characterized by the use of an aldehydebased chemical instead of osmium tetroxide. For more data regarding the Golgi-Kopsch method, please refer to "Reliable Golgi-Kopsch method” (Riley, 1979). Triton X-100 can be added to the Golgi-Kopsch method to improve the staining of specimens with short fixation periods, which are otherwise not well stained (Tokuno et al., 1990).

\section{REPRESENTATIVE IMAGES OF EACH STAINING METHOD}

\section{Golgi-Cox Method}

Fig. 1 shows an adult mouse brain with Golgi-Cox staining, with the various types of cells being well stained. Fig. 1A and B shows images of Golgi-Cox staining of the cerebral cortex, with clearly stained neurons and no noise, such as that from surrounding glial cells. Hippocampal granule cells can be clearly observed in Fig. 1C, and a Purkinje cell is shown in Fig. 1D. Dendritic spines are observed at a high magnification in Fig. 1E.

\section{Rapid Golgi}

Rapid Golgi staining can be performed for various parts of the brain. Experimental results differ depending on the type of nerve tissue in each part. In a mouse brain (cerebral cortex, diencephalon, and other brain stem) stained using the rapid Golgi method, neuronal staining is evident in the order of cortex $>$ brain stem $>$ diencephalon. However, because glial cells and blood vessels are also stained (Fig. 2); thus, careful
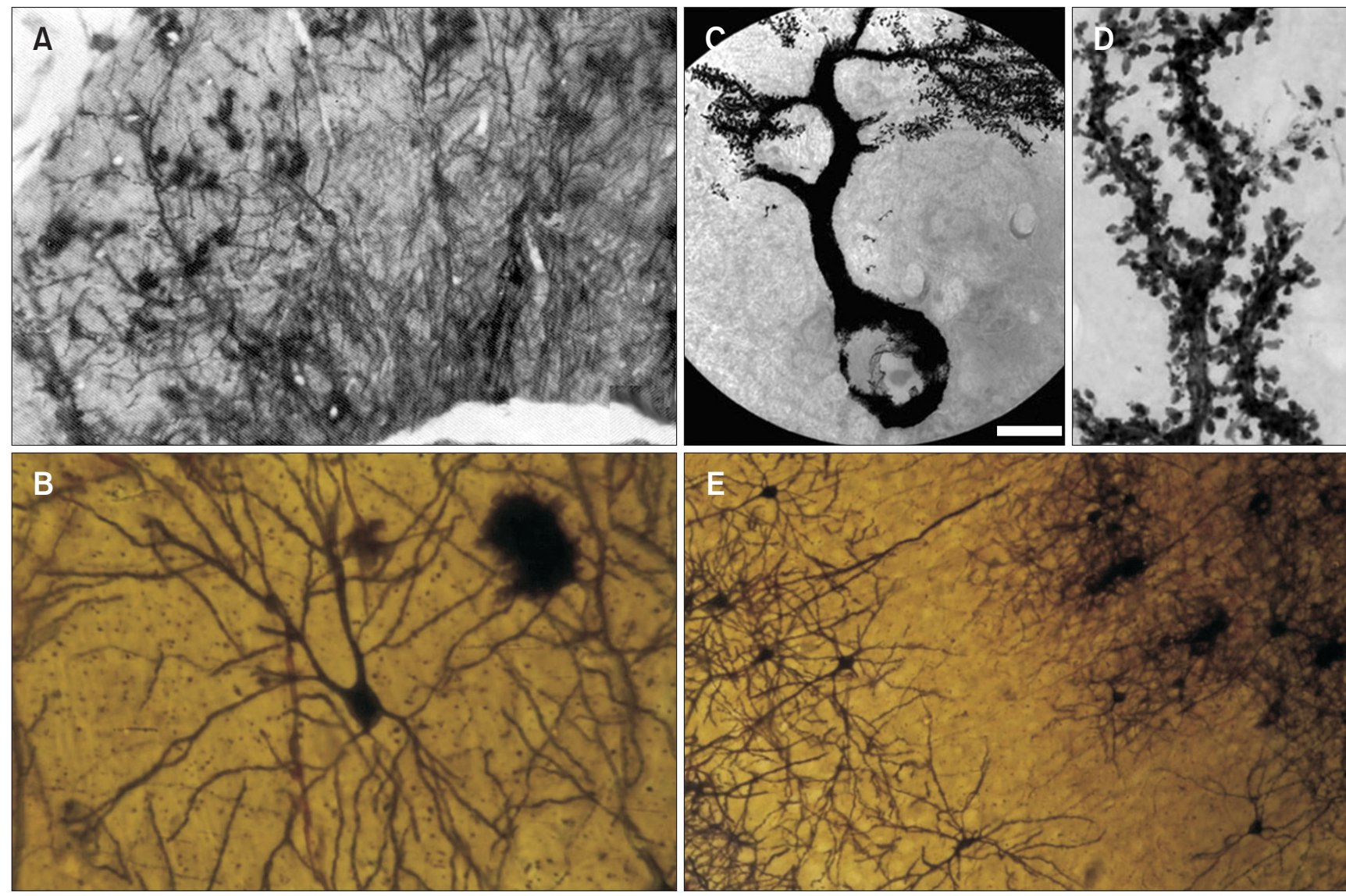

Fig. 2. Adult mouse brain specimen prepared using the rapid Golgi method (from Ahn et al., 1990; Oda et al., 2010; Rhyu et al., 2016, with kind permission). Hippocampal neurons near the neuroglia and blood vessel were labelled (A). A neuron and glial cell in the hilus of the dentate gyrus (B). An image of a Purkinje cell and its dendritic spines taken using a high-voltage electron microscope $(\mathrm{C}, \mathrm{D})$ and pyramidal neurons in the motor cortex (E). 
interpretation is required. High-voltage electron microscopy combined with rapid Golgi staining can show clear neuronal cytology, including the cell body, axon, dendrites, and dendritic spines (Fig. 2C and D; Oda et al., 2010). Recent application of pan-focal 3D Edge microscopy yields a finer image by overcoming the limitation of focal depth (Fig. 2B and E; Rhyu et al, 2016).

\section{Golgi-Kopsch Method}

The Golgi-Kopsch method is one of the most effective methods for staining extensively fixed specimens. Fig. 3 shows images of rat brain specimens that are formalin fixed for 1 month and stained using the Golgi-Kopsch method. Neurons, neuroglial cells, and blood vessels are observed in the pyriform cortex (Fig. 3A), thalamus (Fig. 3B), caudate putamen (Fig. 3C), and reticular formation (Fig. 3D). The neuronal body, axon, and dendrites can be clearly visualized, but tiny structures such as dendritic spines are not clear. The Golgi-Kopsch method can efficiently label long-termfixed brains, up to 50 years after fixation, although it does not provide good labeling of fresh fixed brains (Rosoklija et al., 2003). To enhance Golgi-Kopsch staining, Triton X-100 can be used during the process. Triton X-100 is effective for the penetration of potassium dichromate and silver nitrate in Golgi staining, thereby labeling neurons strongly and revealing small structures such as dendritic spines (Tokuno et al., 1990).

\section{SUGGESTION FOR AN APPROPRIATE GOLGI STAINING METHOD}

\section{Golgi-Cox Method}

The Golgi-Cox method is the primary method for ensuring the staining of entire dendritic trees of cortical neurons. It is also the best for analyzing complete dendritic trees of cortical neurons because the background is less dense than that obtained using the rapid Golgi method. It is an optimal method for examining different types of neurons in the adult brain (Buell, 1982). Compared with the other two staining methods, the Golgi-Cox method has the advantage of clearly staining dendritic spines and the cell body.

However, the Golgi-Cox method has some limitations. First, specimens stained with the Golgi-Cox method tend to shrink more than those stained with the rapid Golgi method. Furthermore, it is not a good option for studying subcortical structures because of its low permeability to dendritic spines of that region (Koyama, 2013). The method is also more complicated than the rapid Golgi method, and the staining is unstable. In addition, similar to the other Golgi staining methods, there is the disadvantage that neuronal cells are nonspecifically stained.

The Golgi-Cox method is still a developing and well-used staining method and is one of the most widely used methods for fresh specimens (Koyama, 2013; Koyama \& Tohyama, 2012). It is the optimal Golgi method for staining cortical areas. Because the background noise is low, it is better to use this staining method to clearly observe dendritic spines and cell bodies.

Several methods have been recently attempted to complement the nonspecific Golgi-Cox method, one of which is the simultaneous Golgi-Cox and immunofluorescence method by Spiga et al. (2011) (Koyama \& Tohyama, 2013). By simultaneously staining brains using immunofluorescence and the Golgi-Cox method, neurons can be observed by targeting specific areas. It is expected this simultaneous immunohistochemistry and Golgi-Cox method will yield
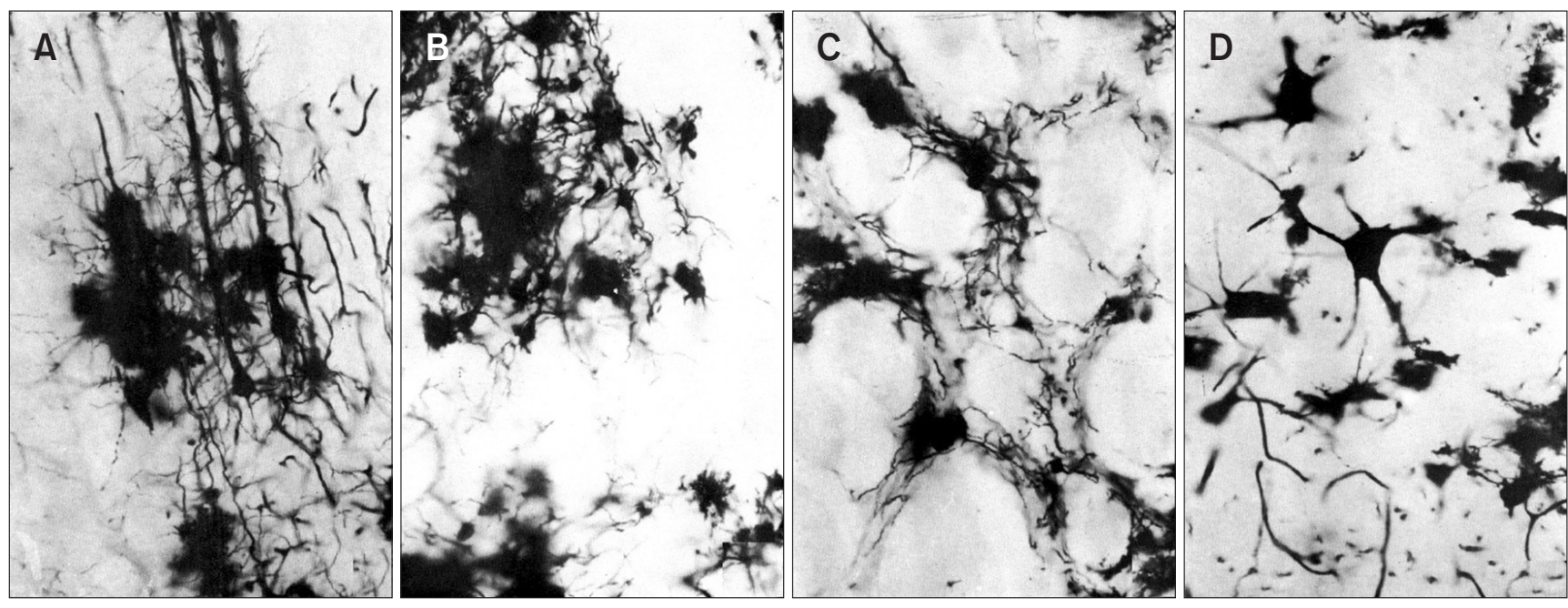

Fig. 3. Neurons labeled using the Golgi-Kopsch method (modified from Anh et al., 1990, with kind permission). Neurons, neuroglia, and blood vessels are labelled in the pyriform cortex (A), thalamus (B), caudate and putamen (C), and the reticular formation (D). 
finer morphological details with neurochemical identity of the nerve cells than staining with the Golgi-Cox method alone.

\section{Rapid Golgi}

The advantages of the rapid Golgi method are that it is easier to stain neuronal cells for anatomical neuroscience research because it is easy to observe the cell body and the axons, dendrites, and dendrites. Among these structures, the axon is particularly well observed using the rapid Golgi method. It is the most suitable for observing detailed anatomical morphology of neurons because it stains finer details of single neurons compared with other staining methods (Ahn et al., 1990).

However, the rapid Golgi method has a disadvantage that the results are sporadic and relatively nonspecific, similar to the other Golgi methods. It stains only $1 \% \sim 10 \%$ of neurons in the brain, with glial cells and surrounding blood vessels also being stained; the reproducibility is also somewhat low. Finally, there is an unfavorable and variable problem with the rapid Golgi method; namely, individual differences between researchers are quite remarkable (Ahn et al., 1990; GarínAguilar et al., 2012).

Although many advantages and disadvantages have been presented, it is a good staining method for anatomical research. Therefore, if procedures can be developed to minimize the abovementioned disadvantages of nonspecific and sporadic staining and to stabilize impregnation, morphological studies of neuronal structures will improve using the rapid Golgi staining method.

\section{Golgi-Kopsch Method}

A characteristic of the Golgi-Kopsch method is that an insufficient number of neurons are impregnated in shortterm-fixed specimens or specimens immediately after perfusion, but the neurons are efficiently impregnated in long-term-fixed specimens. However, the inclusion of Triton X-100 in the staining process effectively increases the number of impregnated neurons. This process is effective for specimens after $>15$ months of fixation. The Golgi-Kopsch method impregnates more neurons and more distinctly stains individual dendritic spines than other Golgi staining methods for specimens that have been fixed for $>15$ months. In addition, when high concentrations of Triton X-100 are used for a long period of time, the number of impregnated neurons increases, but dendritic spines and trees are not as clearly observed; hence, caution must be taken while determining how to use Triton X-100.

Therefore, the Golgi-Kopsch method is suitable for cases in which human brains have been fixed for at least 15 months or specimens have been obtained from old-aged animals, rather than for specimens that need to be observed right after extraction or perfusion (Koyama, 2013). However, the modified Golgi-Kopsch method using Triton X-100 is suitable for staining specimens that have been fixed for $<15$ months, as well as for those that have been fixed for $\geq 15$ months, if the purpose of observation is on a global scale rather than at an individual neuron level.

\section{CONCLUSIONS}

This study describes Golgi staining and broadly reviews the Golgi-Cox, rapid Golgi, and Golgi-Kopsch staining methods. It also outlines methodological differences and the advantages and disadvantages of the three Golgi methods. The comparison of the results of these staining methods will help researchers in selectin the type of Golgi method suitable for their study purpose or for specific specimens.

Until now, the difficulties of targeting specific cells and the complexity of the procedures have led researchers to avoid using the Golgi method. However, as massive amounts of data using Golgi methods have accumulated since the invention of the method and as it is currently possible to use technologies such as immunofluorescence along with the Golgi method to provide complete and clear views of neuronal structures, the Golgi method will be a fascinating tool for future analyses of normal and pathologic states of neurons.

\section{CONFLICT OF INTEREST}

No potential conflict of interest relevant to this article was reported.

\section{REFERENCES}

Ahn B K, Park M J, Kim E H, Bae Y C, Hong H S, Cho H J, and Joo K (1990) A study on staining techniques of Golgi methods. Kyungpook Univ. Med. J. 31, 313-322.

Bentivoglio M, Jones E G, Mazzarello P, Ribak C E, Shepherd G M, and Swanson L W (2011) Camillo Golgi and modern neuroscience. Brain Res. Rev. 66, 1-4.

Buell S J (1982) Golgi-Cox and rapid golgi methods as applied to autopsied human brain tissue: widely disparate results. J. Neuropathol. Exp. Neurol. 41, 500-507.

De Carlos J A and Borrell J (2007) A historical reflection of the contributions of Cajal and Golgi to the foundations of neuroscience. Brain Res. Rev. 55, 8-16.

Garín-Aguilar M E, Díaz-Cintra S, Quirarte G L, Aguilar-Vázquez A, Medina A C, and Prado-Alcalá R A (2012) Extinction procedure induces 
pruning of dendritic spines in CA1 hippocampal field depending on strength of training in rats. Front. Behav. Neurosci. 6, 12.

Glickstein M (2006) Golgi and Cajal: the neuron doctrine and the 100th anniversary of the 1906 Nobel Prize. Curr. Biol. 16, R147-R151.

Golgi C (1873) On the structure of the brain grey matter. Gazzetta Medica Italiana. Lombardia 33, 244-246.

Jones E G (2011) Cajal's debt to Golgi. Brain Res. Rev. 66, 83-91.

Koyama Y (2013) The unending fascination with the Golgi method. OA Anatomy 1, 8.

Koyama Y and Tohyama M (2012) A modified and highly sensitive Golgi-Cox method to enable complete and stable impregnation of embryonic neurons. J. Neurosci. Methods 209, 58-61.

Koyama Y and Tohyama M (2013) A novel, Golgi-Cox-based fluorescent staining method for visualizing full-length processes in primary rat neurons. Neurochem. Int. 63, 35-41.

Maiti P, Manna J, and McDonald M P (2015) Merging advanced technologies with classical methods to uncover dendritic spine dynamics: A hot spot of synaptic plasticity. Neurosci. Res. 96, 1-13.

Narayanan S N, Jetti R, Gorantla V R, Kumar R S, Nayak S, and Bhat P $G$ (2014) Appraisal of the effect of brain impregnation duration on neuronal staining and morphology in a modified Golgi-Cox method. J. Neurosci. Methods 235, 193-207.

Oda S I, Lee KJ, Arii T, Imoto K, Hyun B H, Park I J, Kim H, and Rhyu I J (2010) Differential regulation of Purkinje cell dendritic spines in rolling mouse Nagoya (tgrol/tgrol), P/Q type calcium channel $(\alpha 1 \mathrm{~A} /$ Cav2.1) mutant. Anat. Cell Biol. 43, 211-217.

Pannese E (1999) The Golgi Stain: invention, diffusion and impact on neurosciences. J. Hist. Neurosci. 8, 132-140.

Pannese E (2007) The contribution of Camillo Golgi to our understanding of the structure of the nervous system. Arch. Ital. Biol. 145, 111-115.
Patro N, Kumar K, and Patro I (2013) Quick Golgi method: modified for high clarity and better neuronal anatomy. Indian J. Exp. Biol. 51, 685693.

Ramon-Moliner E (1970) The Golgi-Cox technique. In: Contemporary Research Methods in Neuroanatomy, ed. Nauta W J H and Ebbesson S O E, pp. 32-55, (Springer-Verlag, New York).

Rhyu Y S, Lee S J, Kim D H, and Uhm C S (2016) Application of threedimensional light microscopy for thick specimen studies. Appl. Microsc. 46, 93-99.

Riley J N (1979) A reliable Golgi-Kopsch modification. Brain Res. Bull. 4, 127-129.

Rosoklija G, Mancevski B, Ilievski B, Perera T, Lisanby S H, Coplan J D, Duma A, Serafimova T, and Dwork AJ (2003) Optimization of Golgi methods for impregnation of brain tissue from humans and monkeys. J. Neurosci. Methods 131, 1-7.

Scheibel M E and Scheibel A R (1978) The Methods of Golgi. In: Neuroanantomical Resarch Techniques, pp. 89-114, (Academic Press, New York).

Smitha J and Roopa R (2012) Rapid Golgi Technique for staining pyramidal neurons in rat hippocampus. IJBAMS 2, 98-102.

Spiga S, Acquas E, Puddu M C, Mulas G, Lintas A, and Diana M (2011) Simultaneous Golgi-Cox and immunofluorescence using confocal microscopy. Brain Struct. Funct. 216, 171-182.

Tokuno H, Nakamura Y, Kudo M, and Kitao Y (1990) Effect of Triton X-100 in the Golgi-Kopsch method. J. Neurosci. Methods 35, 75-77.

Valverde F (1970) The Golgi Method. A Tool for comparative Structural Analyses (Springer, New York).

Zaqout S and Kaindl A M (2016) Golgi-Cox staining step by step. Front. Neuroanat. 10, 38 